\title{
HPLC: Highly Accessible Instrument in Pharmaceutical Industry for Effective Method Development
}

\section{Atanu Kumar Jena*}

Department of Biotechnology, Dr. M.G.R Educational \& Research Institute, Dr. M.G.R University, Chennai

\begin{abstract}
The analytical technique of High Performance Liquid Chromatography (HPLC) is used extensively thought the pharmaceutical industry. It allows simultaneously both qualitative and quantitative information of a drug. It is used to provide information on the composition of drug related samples. The information obtained may be qualitative, indicating what compounds are present in the sample. The information obtained may be qualitative, providing the actual amount of compounds in the sample. HPLC is used in all the different stages in the creation of new drug discoveries and also used routinely in the drug manufacturing process. The HPLC techniques is highly accessible to the author's knowledge this review represents the most exhaust description of instrumentation, method development, recent advancement, area of application. This article reviews the most recent advances in sample preparation, separation and the mass spectrometric aspects of high-throughput for method development.
\end{abstract}

Keywords: High Performance Liquid Chromatography; Method development; Pharmaceutical analysis

\section{Introduction}

High-performance liquid chromatography (sometimes referred to as high-pressure liquid chromatography), HPLC, is a chromatographic technique that can separate a mixture of compounds and is used in biochemistry and analytical chemistry to identify, quantify and purify the individual components of the mixture. HPLC typically utilizes different types of stationary phases, a pump that moves the mobile phase(s) and analyte through the column and a detector to provide a characteristic retention time for the analyte. The detector may also provide additional information related to the analyte, (i.e. UV/Vis spectroscopic data for analyte if so equipped) [1]. Analyte retention time varies depending on the strength of its interactions with the stationary phase, the ratio/composition of solvent(s) used and the flow rate of the mobile phase. It is a form of liquid chromatography that utilizes smaller column size, smaller media inside the column and higher mobile phase pressures [2].

With HPLC, a pump provides the higher pressure required to move the mobile phase and analyte through the densely packed column. The increased density arises from smaller particle sizes. This allows for a better separation on columns of shorter length when compared to ordinary column chromatography. The sample to be analyzed or separated is introduced, in small volumes, into the stream of mobile phase $[3,4]$. The solution moved through the column is slowed by specific chemical or physical interactions with the stationary phase present within the column. The velocity of the solution depends on the nature of the sample and on the compositions of the stationary (column) phase. The time at which a specific sample elutes (comes out of the end of the column) is called the retention time; the retention time under particular conditions is considered an identifying characteristic of a given sample $[5,6]$. The use of smaller particle size column packing (which creates higher backpressure) increases the linear velocity giving the components less time to diffuse within the column, improving the chromatogram resolution. Common solvents used include any miscible combination of water or various organic liquids (the most common are methanol and acetonitrile). Water may contain buffers or salts to assist in the separation of the sample components, or compounds such as trifluoroacetic acid which acts as an ion pairing agent [7].

\section{History of HPLC}

Liquid chromatography was initially discovered as an analytical technique in the early twentieth century and was first used as a method of separating colored compounds. This is where the name chromatography, chroma means color, graphy means writing, was derived [8]. A Russian botanist used a rudimentary form of chromatographic separation to purify mixtures of plant pigments into the pure constituents. He separated the pigments based on their interaction with a stationary phase, which is essential to any chromatographic separation. The stationary phase he used was powdered chalk and alumina, the mobile phase in his separation was the solvent. After the solid stationary phase was packed into a glass column (essentially a long, hollow, glass tube) he poured the mixture of plant pigments and solvent in the top of the column. He then poured additional solvent into the column until the samples were eluted at the bottom of the column $[9,10]$. The result of this process most crucial to his investigation was that the plant pigments separated into bands of pure components as they passed through the stationary phase. Modern high performance liquid chromatography or HPLC has its roots in this separation, the first form of liquid chromatography. The chromatographic process has been significantly improved over the last hundred years, yielding greater separation efficiency, versatility and speed.

\section{Instrumentation of HPLC}

Instrumentation is required to enable the follow of the mobile phase through the stationary phase and also to convert the separated

*Corresponding author: Atanu Kumar Jena, Department of Biotechnology, Dr. M.G.R Educational \& Research Institute, Dr. M.G.R University,Chennai-600095, India, E-mail: atanujena_1987@yahoo.co.in

Received January 01, 2012; Accepted January 25, 2012; Published January 27, 2012

Citation: Atanu Kumar J (2012) HPLC: Highly Accessible Instrument in Pharmaceutical Industry for Effective Method Development. Pharm Anal Acta 3:147. doi:10.4172/2153-2435.1000147

Copyright: @ 2012 Atanu Kumar J. This is an open-access article distributed unde the terms of the Creative Commons Attribution License, which permits unrestricted use, distribution, and reproduction in any medium, provided the original author and source are credited. 
component into meaning full information (Figure 1). The HPLC system is very complicated system it consist of different components given in the (Table 1,2).

\section{Mobile phase reservoir}

The mobile phase usually a glass containers. The containers need to be of an appropriate size so that it can contain enough mobile phase for the analysis being performed. PTFE tubing connects the content of the reservoir with the HPLC system. This tubing is typically of outer diameter (OD) $1 / 8$ inches and the inner diameter is (ID) $1 / 16$ inch. The size of the tubing in the HPLC system usually measured using the empirical system of inches. At the end of the tubing which is contact with the mobile phase there is usually a filter $(10 \mu \mathrm{m})$ to remove any particulate matter, this is also act as a 'sinker' to hold the tubing at the bottom of the container. This is commonly glass, stainless steel. A lid to the container needs to allow a space for tubing, purpose made lid can be purchased [11]. It is important not to seal the reservoir tightly to avoid the creation of vacuum. The number of lines available on the instrumentation to perform reversed phase gradient elution more than one line is required so that the proportion of the organic component

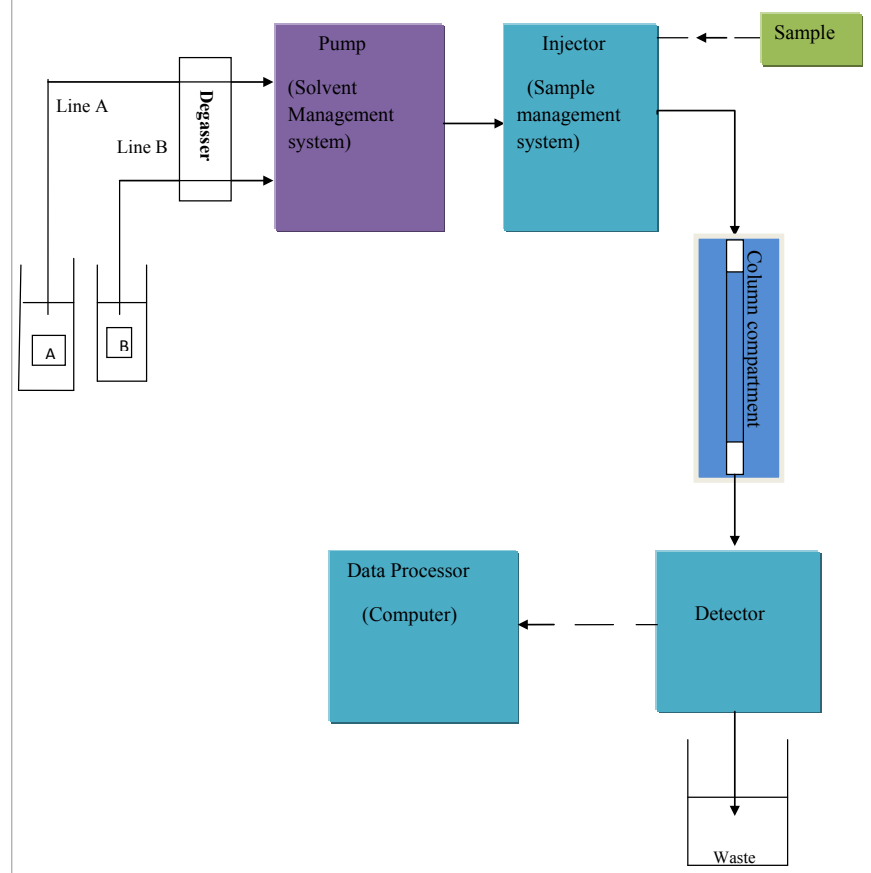

Figure 1: Configuration of typical HPLC System

\begin{tabular}{|c|c|}
\hline HPLC system component & Description \\
\hline Mobile phase reservoir & Stock of mobile phase for required analysis \\
\hline Degasser & Degasses the mobile phase \\
\hline Pump & $\begin{array}{l}\text { Solvent delivery system, enable the follow of mobile } \\
\text { phase }\end{array}$ \\
\hline Sample valve Injector & $\begin{array}{l}\text { Sample delivery system, Introduces the sample to } \\
\text { the system }\end{array}$ \\
\hline Column Component & Used to control the temperature of the column \\
\hline Detector & $\begin{array}{l}\text { Detect each component in the mixture for ,after } \\
\text { eluted from column }\end{array}$ \\
\hline Data Acquisition & $\begin{array}{l}\text { Convert the date from the detector to a meaning full } \\
\text { result }\end{array}$ \\
\hline Waste & Collection of liquid waste \\
\hline
\end{tabular}

in the mobile phase can be increased throughout the analysis. For isocratic (1-line is available), binary (2-lines are available), ternary (3 lines are available).

\section{Degassing}

In HPLC degassing of solvents, mobile phase is very essential for proper operation and retrieval of uniform data. Degassing removes entrapped air bubbles from the solvents. Improper degassing leads to many problems like fluctuation in pressure, improper peaks, improper detection, clogging of column etc. Degassing is done by degasser techniques like ultra sonication, filtration etc. Ultrasonication Here gas is removed by either bursting of gas bubbles immersing ultra sonic generator into the solvent or by placing the solvent container into ultra sonicator tray $[12,13]$. This is the commonest technique for degassing of HPLC solvents.

\section{Pump}

Because of the small particles used in modern HPLC, modern LC pumps need to operate reliably and precisely at pressures of 10,000 p.s.i. or at least 6,000 p.s.i. To operate at these pressures and remain sensibly inert to the wide variety of solvents used HPLC pumps usually have sapphire pistons, stainless steel cylinders and return valves fitted with sapphire balls and stainless steel seats. For analytical proposes HPLC pumps should have flow rates that range from 0 to $10 \mathrm{ml} / \mathrm{min}$., but for preparative HPLC, flow rates in excess of $100 \mathrm{ml} / \mathrm{min}$ may be required. It is extremely difficult to provide a very constant flow rate at very low flow rates [14]. If .1\% is considered acceptable then for $100 \mathrm{uls} / \mathrm{min}$ a flow variation of less then $.1 \mathrm{ul} / \mathrm{min}$ is required. This level of constancy is required because most HPLC detectors are flow sensitive and errors in quantization will result from changes in flow rate [15].

\section{Sample valve injector}

Since sample valves come between the pump and the column it follows that HPLC sample valves must also tolerate pressures up to 10,000 p.s.i. For analytical HPLC, the sample volume should be selectable from sub-micro liter to a few micro liters, whereas in preparative HPLC the sample volume may be even greater than $10 \mathrm{ml}$. To maintain system efficiency the sample valve must be designed to have very low dispersion characteristics, this is true not only for flow dispersion but also for the less obvious problems of dispersion caused by sample adsorption/desorption on valve surfaces and diffusion of sample into and out of the mating surfaces between valve moving parts $[16,17]$. It goes without saying that the valves must deliver a very constant sample size but this is usually attained by the use of a constant size sample loop.

\section{Column component}

HPLC columns are packed with very fine particles (usually a few microns in diameter). The very fine particles are required to attain the low dispersion that give the high plate counts expected of modern HPLC. Plate counts in excess of 25,000 plates per column are possible with modern columns, however, these very high efficiencies are very rarely found with real samples because of the dispersion associated with injection valves, detectors, data acquisition systems and the dispersion due to the higher molecular weight of real samples as opposed to the common test samples. Packing these small particles into the column is a difficult technical problem but even with good packing a great amount of care must be given to the column end fittings and the inlet and outlet connection to keep dispersion to a minimum [18]. Some 


\begin{tabular}{|c|c|c|c|c|}
\hline TYPE & SAMPLE POLARITY & $\begin{array}{c}\text { MOLECULAR } \\
\text { WEIGHT RANGE }\end{array}$ & STATIONARY PHASE & MOBILE PHASE \\
\hline Adsorption & non-polar to somewhat polar & $10^{0}-10^{4}$ & silica or alumina & non-polar to polar \\
\hline Partition (reversed-phase) & non-polar to somewhat polar & $10^{0}-10^{4}$ & $\begin{array}{l}\text { non-polar liquid adsorbed or chemically bonded to the } \\
\text { packing material }\end{array}$ & relatively polar \\
\hline Partition (normal-phase) & somewhat polar to highly polar & $10^{0}-10^{4}$ & $\begin{array}{l}\text { highly polar liquid adsorbed or chemically bonded to the } \\
\text { packing material }\end{array}$ & relatively non-polar \\
\hline Ion Exchange & highly polar to ionic & $10^{0}-10^{4}$ & $\begin{array}{l}\text { ion-exchange resins made of insoluble, high-molecular } \\
\text { weight solids functionalized typically with sulfonic acid } \\
\text { (cationic exchange) or amine (anionic exchange) groups }\end{array}$ & $\begin{array}{l}\text { aqueous buffers with added } \\
\text { organic solvents to moderate } \\
\text { solvent strength }\end{array}$ \\
\hline Size-Exclusion & non-polar to ionic & $10^{3}-10^{6}$ & small, porous, silica or polymeric particles & polar to non-polar \\
\hline
\end{tabular}

Table 2: Different type of HPLC which are used for Method development for Pharmaceutical industries

state of the art systems are now 'chip' based and may use no particles at all. Some limited use has been made of HPLC for preparative purposes using half inch to one inch diameter columns. LC columns, in general, achieve their separation by exploiting the different intermolecular forces between the solute and the stationary phase and those between the solute and the mobile phase [19]. The column will retain those substances that interact more strongly with the stationary phase than those that interact more strongly with the mobile phase. The basic intermolecular forces that are exploited in the HPLC are the same as those discussed in The Mechanism of Chromatographic Retention and The Thermodynamics of Chromatography of the Chrom-Ed series. The main consideration with HPLC is the much wider variety of solvents and packing materials that can be utilized as because of the much lower quantities of both which are required. In particular very expensive optically pure compounds can be used to make Chiral HPLC stationary phases and may even be used as (disposable) HPLC solvents [20].

\section{Detector}

LC detectors have been extensively discussed in Liquid Chromatography Detectors and HPLC detectors use the same detection principals with extra care being given to the small solute elution volumes that result from the combination of high column efficiencies with small volumes. In order to give an accurate chromatographic profile the detector sampling (cell) volume must be a small fraction of the solute elution volume. If the detector volume were larger than the elution volume then you would have peaks that appeared with flat tops as the whole peak would be resident in the detector at the same time. This means that as column volumes decrease and system efficiencies increase the volume of the detector cell volume must also decrease $[21,22]$. This is of course at odds for the requirement for detector to maintain high sensitivity as this is usually dependant on having a larger cell volume. Again, this requires the very careful design of modern detectors (Table 3).

\section{Data acquisition}

Data acquisition was discussed in Liquid Chromatography Detectors and the only extra consideration required for HPLC is the higher sampling rate needed for the rapidly eluting narrow peaks of the HPLC chromatogram. Although the theoretical number of samples needed for good quantization are actually quite small, for real systems a hundred samples or more per peak is recommended; thus, for a $4 \mathrm{sec}$ wide peak, a rate of 25 samples per second may be required. The same data analysis and reporting software can be used as in ordinary LC [23].

\section{Recent Advancement in HPLC}

Recent advances in both ionization methods and mass spectrometers have resulted in powerful new techniques for the study of drug metabolism and disposition. The interest in high-performance liquid chromatography/mass spectrometry (HPLC/MS) is the result of the lack of a sensitive universal detector for HPLC. Although it is not the ideal detector, HPLC/MS has become a reliable technique for xenobiotic analysis. The application of HPLC/MS to studies of the pharmacology and toxicology of molecules of mass $<1,500$ daltons is most advantageous in three areas: development of specific methods for trace analysis, detection and characterization of metabolites and studies of interactions between drug molecules and peptides/proteins. We have used HPLC/MS to study the deposition of cyclosporine and its metabolites in needle biopsy samples from kidney and liver in which sample size is severely limited.[24,25]The limit of detection in the single-ion monitoring mode was $500 \mathrm{fg}$ ( $450 \mathrm{amol})$, which is about a thousand fold lower than UV limits of detection.

UPLC refers to Ultra Performance Liquid Chromatography. It improves in three areas: chromatographic resolution, speed and sensitivity analysis. It uses fine particles and saves time and reduces solvent consumption. UPLC is comes from HPLC. HPLC has been the evolution of the packing materials used to effect the separation. An underlying principle of HPLC dictates that as column packing particle size decreases, efficiency and thus resolution also increases. As particle size decreases to less than $2.5 \mu \mathrm{m}$, there is a significant gain in efficiency and it's doesn't diminish at increased linear velocities or flow rates according to the common Van Deemter equation. By using smaller particles, speed and peak capacity (number of peaks resolved per unit time) can be extended to new limits which is known as Ultra Performance [26,27]. The classic separation method is of HPLC (High Performance Liquid Chromatography) with many advantages like robustness, ease of use, good selectivity and adjustable sensitivity. Its main limitation is the lack of efficiency compared to gas chromatography or the capillary electrophoresis due to low diffusion coefficients in liquid phase, involving slow diffusion of analytes in the stationary phase. The Van Deemter equation shows that efficiency increases with the use of smaller size particles but this leads to a rapid increase in back pressure, while most of the HPLC system can operate only up to 400 bar. That is why short columns filled with particles of about $2 \mu \mathrm{m}$ are used with these systems, to accelerate the analysis without loss of efficiency, while maintaining an acceptable loss of load [28].

Liquid chromatography-mass spectrometry (LC-MS, or alternatively HPLC-MS) is an analytical chemistry technique that combines the physical separation capabilities of liquid chromatography (or HPLC) with the mass analysis capabilities of mass spectrometry. LC-MS is a powerful technique used for many applications which has very high sensitivity and selectivity [29]. Generally its application is oriented towards the general detection and potential identification of chemicals in the presence of other chemicals (in a complex mixture). The limitations of LC-MS in urine analysis drug screening are that it often fails to distinguish between specific metabolites, in particular with 


\begin{tabular}{|c|c|c|c|}
\hline Detector & Analytes & Solvent Requirement & Comments \\
\hline UV-vis & Any with chromophores & UV-grade, non-UV absorbing solvent & $\begin{array}{l}\text { Has a degree of selectivity and is useful } \\
\text { for many HPLC Application }\end{array}$ \\
\hline Fluorescence & Fluorescent compound & UV-grade, non-UV absorbing solvent & $\begin{array}{l}\text { Highly selective and sensitive. Often used } \\
\text { to analyzed derivatized compounds }\end{array}$ \\
\hline Refractive Index (Ri) & $\begin{array}{l}\text { Compound with different RI to mobile } \\
\text { Phase }\end{array}$ & Cannot run mobile phase gradient & $\begin{array}{l}\text { Virtually a universal detector but has } \\
\text { limited sensitivity }\end{array}$ \\
\hline Conductivity & Charged or polar compound & Mobile phase must be Conducting & Excellent for ion Exchange method \\
\hline Electrochemical & $\begin{array}{l}\text { Readily oxidized or reduced compounds } \\
\text { Especially biological sample }\end{array}$ & Mobile phase must be conducting & Very selective \& sensitive \\
\hline Evaporative light Scattering (ELSD) & Virtually all compound & $\begin{array}{l}\text { Must use volatile solvents and volatile } \\
\text { buffers }\end{array}$ & $\begin{array}{l}\text { A universal detector which is highly } \\
\text { sensitive. Not selective }\end{array}$ \\
\hline Mass spectrometer (MS) & Broad range of compound & Must use volatile solvent $\&$ volatile buffers & $\begin{array}{l}\text { Highly sensitive \& powerful } 2^{\text {nd }} \text { dimension } \\
\text { analytical tool }\end{array}$ \\
\hline
\end{tabular}

Table 3: Different type of detector \& which require suitable solvent for the detection of Analytes

hydrocarbon and its metabolites. LC-MS urine analysis testing is used to detect specific categories of drugs however Gas chromatography (GC-MS) should be used when detection of a specific drug and its metabolites is required [30].

Liquid chromatography linked to tandem mass spectrometry (LC-MS/MS) has played an important role in pharmacokinetics and metabolism studies at various drug development stages since its introduction to the pharmaceutical industry. Newly introduced techniques such as ultra-performance liquid chromatography with small particles (sub-2 $\mu \mathrm{m}$ ) and monolithic chromatography offer improvements in speed, resolution and sensitivity compared to conventional chromatographic techniques. Hydrophilic interaction chromatography (HILIC) on silica columns with low aqueous/high organic mobile phase is emerging as a valuable supplement to the reversed-phase LC-MS/MS. Sample preparation formatted to 96-well plates has allowed for semi-automation of off-line sample preparation techniques, significantly impacting throughput [31]. On-line solidphase extraction (SPE) utilizing column-switching techniques is rapidly gaining acceptance in bioanalytical applications to reduce both time and labor required to produce bioanalytical results. Extraction sorbents for on-line SPE extend to an array of media including large particles for turbulent flow chromatography, restricted access materials (RAM), monolithic materials and disposable cartridges utilizing traditional packing's such as those used in Spark Holland systems. In the end, this paper also discusses recent studies of matrix effect in LCMS/MS analysis and how to reduce/eliminate matrix effect in method development and validation [32-34].

\section{HPLC in Different Area of Pharmaceutical}

HPLC can be used in both qualitative and quantitative applications that are for both compound identification and quantification. Normal phase HPLC is only rarely used now, almost all HPLC separation can be performed in reverse phase. Reverse phase HPLC (RPLC) is ineffective in for only a few separation types; it cannot separate inorganic ions (they can be separated by ion exchange chromatography). It cannot separate polysaccharides (they are too hydrophilic for any solid phase adsorption to occur), nor poly-nucleotides (they adsorb irreversibly to the reverse phase packing). Lastly, incredibly hydrophobic compounds cannot be separated effectively by RPLC (there is little selectivity). Aside from these few exceptions, RPLC is used for the separation of almost all other compound varieties. RPLC can be used to effectively separate similar simple and aromatic hydrocarbons, even those that differ only by a single methylene group. RPLC effectively separates simple amines, sugars, lipids and even pharmaceutically active compounds [35]. RPLC is also used in the separation of amino acids, peptides and proteins. Finally RPLC is used to separate molecules of biological origin. The determination of caffeine content in coffee products is routinely done by RPLC in commercial applications in order to guarantee purity and quality of ground coffee. HPLC is a useful addition to an analytical arsenal, especially for the separation of a sample before further analysis.

\section{Specialty Chemical Analysis}

The development stage, where HPLC is used to characterize products of the chemical synthesis, by analyzing the active pharmaceutical ingredients (API), their impurities and/or degradation products generated by accelerated aging. The development of formulation requires also studies of the dissolution properties of solid dosage forms as well as assays of the pharmaceutical formulations. Method for the verification of system's cleanliness during the manufacturing process are developed and used at this stage [36,37]. All the HPLC methods that have been finalized at the developmental stage are validated and transferred to the manufacturing laboratories for a quality control analysis

In-process control method monitors the progress in the manufacturing of an active pharmaceutical ingredient or its formulation. HPLC is frequently employed for the in-process assay of active pharmaceutical ingredient synthesis. The results signal the production chemist/pharmacist whether to proceed with a subsequent unit operation. The decision about whether to use an inprocess control test in a manufacturing is established during process development and is based on scientific judgment. In recent years the FDA issued recommendations for process analytical technology (PAT) [38]. The guidance intended to describe a regulatory framework that will encourage the voluntary development and implementation of innovative pharmaceutical development, manufacturing and quality assurance [39]. The new LC ultra performance/rapid technologies are emerging as the technology of choice for the in-process control assay due to their extended efficiency, sensitivity and speed of the analysis.

\section{Pharmaceutical Analysis}

To understand the purpose of HPLC analytical method it is necessary to consider the applications of HPLC in pharmaceutical analysis There are wide verity of application throughout the processing of new drugs, from the initial drug discovery to manufacture of formulated products which will administered to the patients [40-42]. In the majority of cases the use of reversed phase (RP) HPLC conditions and UV detection has been reported. Several general studies support the selection of the optimal chromatographic conditions investigated 
the orthogonality of 38 chromatographic systems with different stationary and mobile phases with different organic modifiers, $\mathrm{pH}$ and column temperature using 68 drug substances as model compounds. The same group described a method for screening RP-HPLC columns (27 different brands) often used in impurity profiling studies based on selectivity differences and overall separation performances. The widely known Dry lab system is often used for the optimization of HPLC systems for impurity profiling [43].

The amount of method development and assay validation required to support analysis of solutions from in vitro systems is a consideration of analytical laboratories performing this type of analysis. As there is little information from regulatory bodies as to how much assay development and validation is required, analytical laboratories need to decide on the best approach for this type of work [44]. In this paper, we describe an efficient 'fit-for-purpose' approach that has been developed to support buffer sample analysis from Safety Pharmacology hERG studies. Results: Method development has been minimized with the aid of compound modeling software and generic HPLC-MS/MS analytical systems. The assay is evaluated prior to sample analysis using simple qualification procedures to support 'one-off' analyses. Conclusion: The result is an efficient process that allows speedy and confident analysis of in vitro samples to successfully support regulatory hERG in vitro studies without the additional workload of a full validation procedure [45].

\section{Pharmaceutical Drug Discovery Analysis}

The goal in the discovery stage of drug development is to discover a new, safe and active chemical entity (NCE) that will become medication for diseases. During the last decade parallel synthesis of potential lead compounds, using combinatorial chemistry has been done. The large numbers of products created by the combinatorial chemistry are then identified by fast LC-MS methods and screened by in-vitro bioassays and/or pharmacological or chemical tests to allow a selection of a few chosen drug candidates [46].

Due to its high sensitivity and selectivity, HPLC coupled with tandem mass spectrometry, HPLC-MS/MS, has become the predominant method in bioassays and pharmacokinetic and metabolic studies. In addition, another new development in this field has been the introduction of column's packing with ultra-fine particles $(<2$ ? $\mathrm{m})$ enabling short columns to be used (5 $\mathrm{cm}$ or less) and rapid analyses (e.g., $5 \mathrm{~min}$ or even less than $1 \mathrm{~min}$ ) to be carried out by UPLC (ultra performance liquid chromatography) [47].

\section{Effective HPLC Method Development}

HPLC analytical method development is a critical process when using HPLC for pharmaceutical analysis. A method needs to separate the desired components satisfactorily, they need to generate the required result and they must be reproducible and robust so that they can be used time after time without problems. The selection of suitable HPLC conditions to achieve a desired separation is often both confusing and time consuming in order to have an efficient method development process, the following three things to keep in mind [48].

The 3 critical components for a HPLC method are: sample preparation, HPLC analysis and standardization (calculations). During the preliminary method development stage, all individual components should be investigated before the final method optimization. This gives the scientist a chance to critically evaluate the method performance in each component and streamline the final method optimization [49].
The rest of the article will discuss the recommended sequence of events and the percentage of time that should be spent on each step in order to meet the method development timeline. One common mistake is that most scientists focus too much on the HPLC chromatographic conditions and neglect the other 2 components of the method (i.e., sample preparation, standardization). The recommended timeline would help scientists investigate different aspects of the method development and allocate appropriate time in all steps [50]. $(10 \%)$

Step 1: Define method objectives and understand the chemistry

Determine the goals for method development (e.g., what is the intended use of the method?) and to understand the chemistry of the analytes and the drug product.

Step 2: Initial HPLC conditions (20\%)

Develop preliminary HPLC conditions to achieve minimally acceptable separations. These HPLC conditions will be used for all subsequent method development experiments.

Step 3: Sample preparation procedure (10\%)

Develop a suitable sample preparation scheme for the drug product.

Step 4: Standardization (10\%)

Determine the appropriate standardization method and the use of relative response factors in calculations.

Step 5: Final method optimization/robustness (20\%)

Identify the "weaknesses" of the method and optimize the method through experimental design. Understand the method performance with different conditions, different instrument set ups and different samples.

\section{Step 6: Method validation (30\%)}

Complete method validation according to ICH guidelines.

\section{Method Objectives}

There is no absolute end to the method development process. The question is what is the "acceptable method performance"? The acceptable method performance is determined by the objectives set in this step. This is one of the most important considerations often overlooked by scientists. In this section, the different end points (i.e., expectations) will be discussed in descending order of significance.

\section{Analytes}

For a related substance method, determining the "significant and relevant" related substances is very critical. With limited experience with the drug product, a good way to determine the significant related substances is to look at the degradation products observed during stress testing. Significant degradation products observed during stress testing should be investigated in the method development. Based on the current ICH guidelines on specifications, the related substances method for active pharmaceutical ingredients (API) should focus on both the API degradation products and synthetic impurities, while the same method for drug products should focus only on the degradation products [51]. In general practice, unless there is any special toxicology concerns, related substances below the limit of quantization (LOQ) should not be reported and therefore should not be investigated.

Significant related substances: Linearity, accuracy and response 
factors should be established for the significant related substances during the method validation. To limit the workload during method development, usually 3 or less significant related substances should be selected in a method [52].

Other related substances: These are potential degradation products that are not significant in amount. The developed HPLC conditions only need to provide good resolution for these related substances to show that they do not exist in significant levels.

\section{Resolution}

A stability indicating method must resolve all significant degradation products from each other. Typically the minimum requirement for baseline resolution is 1.5 . This limit is valid only for 2 Gaussian-shape peaks of equal size. In actual method development, Rs $=2.0$ should be used as a minimum to account for day to day variability, non-ideal peak shapes and differences in peak sizes [53].

\section{Limit of Quantization}

The desired method LOQ is related to the ICH reporting limits. If the corresponding ICH reporting limit is $0.1 \%$, the method LOQ should be $0.05 \%$ or less to ensure the results are accurate up to one decimal place. However, it is of little value to develop a method with an LOQ much below this level in standard practice because when the method is too sensitive, method precision and accuracy are compromised.

\section{Precision, Accuracy}

Expectations for precision and accuracy should be determined on a case by case basis. For a typical related substance method, the RSD of 6 replicates should be less than $10 \%$. Accuracy should be within $70 \%$ to $130 \%$ of theory at the LOQ level [54].

\section{Analysis time}

A run time of about 5-10 minutes per injection is sufficient in most routine related substance analyses. Unless the method is intended to support a high-volume assay, shortening the run time further is not recommended as it may compromise the method performance in other aspects (e.g., specificity, precision and accuracy) [55].

\section{Adaptability for automation}

For methods that are likely to be used in a high sample volume application, it is very important for the method to be "automatable". The manual sample preparation procedure should be easy to perform. This will ensure the sample preparation can be automated in common sample preparation workstations.

\section{Sample solvent selection}

This stage focuses on the selection of the sample solvent (for extraction) and the proper sample preparation procedures. Investigate the effect of sample solvents of different \% organic, $\mathrm{pH}$, extraction volume and extraction procedure on accuracy, precision, sensitivity (LOQ) and the changes in the chromatography (e.g., peak shape, resolution). Whenever possible use the mobile phase in the sample preparation (Figure 2). This will ensure that there will not be any compatibility issues between the sample solution and the HPLC conditions [56].

Accuracy: To investigate the accuracy in sample preparation (i.e., extraction efficiency), prepare a spiked solution by adding known amounts of related substances into a sample matrix. Compare responses of the spike solutions and the neat standard solutions to assess the recovery from the sample preparation. In this stage, since only one particular step is being investigated (i.e., sample preparation), close to theoretical recovery should be observed at this point (e.g., 90$110 \%)$ [57].

Precision: Use the stressed sample to represent the worst case scenario and perform replicate sample preparations from the same sample composite. Investigate the consistency of the related substance profile (i.e., any missing peaks?) and the repeatability results from these preparations [58].

\section{Standardization}

Area \% method: If the response of the active pharmaceutical ingredient is linear from LOQ to the nominal sample concentration, use the $\%$ area approach where the related substance is reported as $\%$ area. This is the most straightforward approach and doesn't require the preparation of standard solutions. It also has the highest precision since preparation to preparation variation will not affect the results. However, in order to ensure the concentration is linear within this range, the sample concentration is usually limited and this will reduce the method sensitivity (i.e., increase LOQ). In general, use this approach as long as the desired LOQ can be achieved $[59,60]$.

External Standard method: Use the external standard method if the response of the active pharmaceutical ingredient is not linear throughout the whole range, or the desired LOQ can't be achieved by the area $\%$ method. The concentration of standard solution should be high enough to ensure the standard solution can be prepared accurately and precisely on a routine basis, it should be low enough to approximate the concentration of related substance in the sample solution. In general, the standard concentration should correspond to about $5 \%$ of related substances [61].

Wavelength Selection and Relative Response Factor: Generate the linearity plot of API and related substances at different wavelengths. At this point, Photodiode Array Detector can be used to investigate the linearity of the active pharmaceutical ingredient and related substances in the proposed concentration range. By comparing the linearity slopes of the active pharmaceutical ingredient and the related substances, one can estimate the relative response factors of the related substances at different wavelengths. The optimum wavelength of detection is the wavelength that gives the highest sensitivity $\left(\lambda_{\max }\right)$ for the significant related substances and minimizes the difference in response factors between those of the active pharmaceutical ingredient and the related substances. After the optimum wavelength is determined, use a highly stressed sample (e.g., 5\% degradation) to verify that the selected wavelength will give the highest $\%$ related substance results $[62,63]$.

\section{Optimization method}

After the individual components of the method are optimized, perform the final optimization of the method to improve the accuracy, precision and LOQ. Use an experimental design approach to determine the experimental factors that have significant impact on the method. This is very important in determining what factors need to be investigated in the robustness testing during the method validation [64]. To streamline the method optimization process, use Plackett Burmann Design (or similar approach) to simultaneously determine the main effects of many experimental factors. Some of the typical experimental factors that need to be investigated are: HPLC conditions: \% organic, $\mathrm{pH}$, flow rate, temperature, wavelength, column age. Sample preparation: \% organic, $\mathrm{pH}$, shaking/sonication, sample size, sample age. Calculation/ standardization: integration, wavelength, standard concentration, response factor correction [65-67]. 


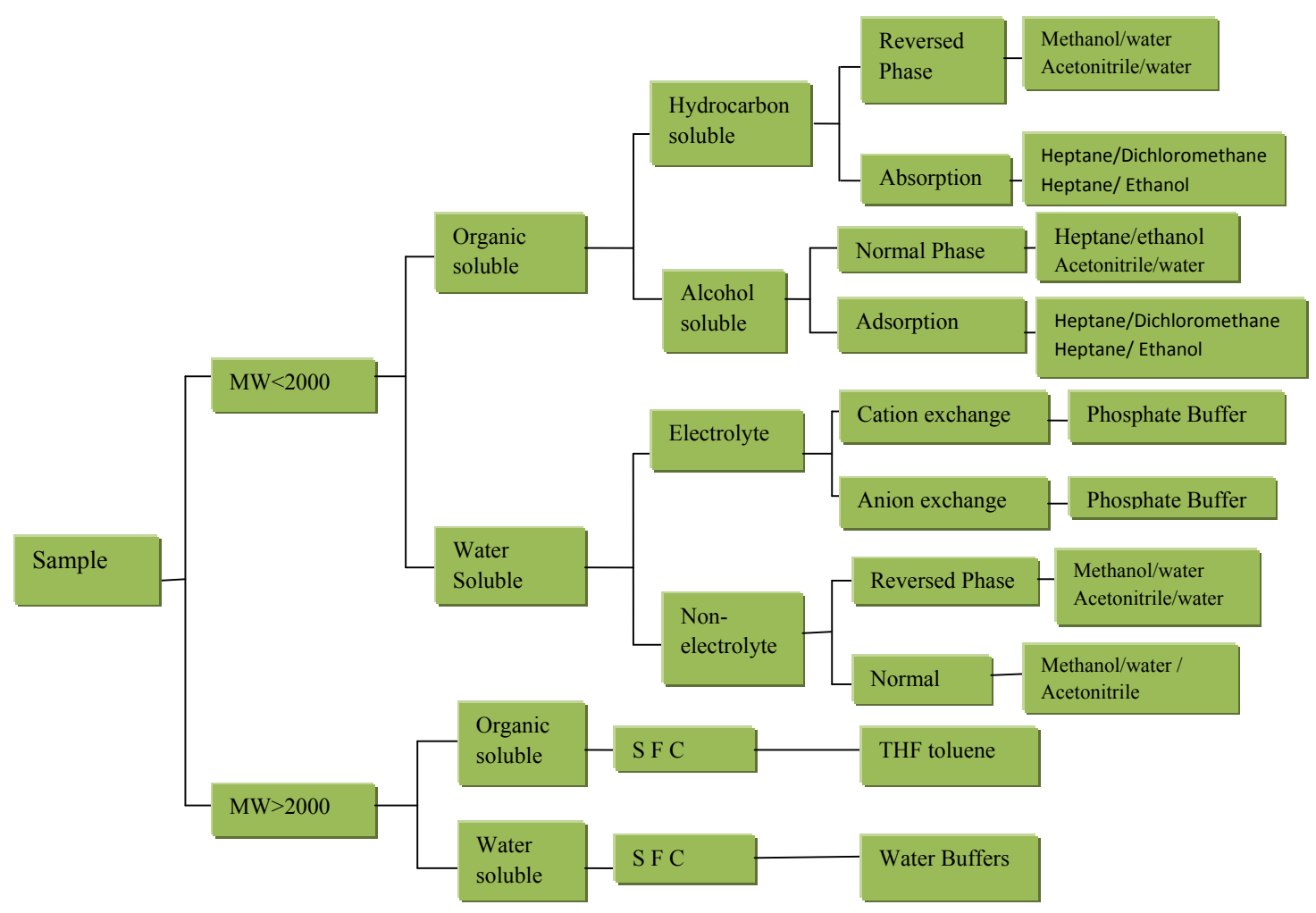

Figure 2: Selection of mobile phase for HPLC.

\section{Validation of HPLC method}

Robustness: Method validation should be treated as a "final verification" of the method performance and should not be used as part of the method development. Some of the typical method validation parameters should be studied thoroughly in the previous steps. In some cases, robustness can be completed in the final method optimization before method validation. At this point, the robustness experiments should be limited only to the most significant factors (usually less than 4 factors) [68-70]. In addition, unlike the final method optimization, the experimental factors should be varied within a narrow range to reflect normal day to day variation. During the method validation, the purpose is to demonstrate that the method performance will not be significantly impacted by slight variations of the method conditions.

Linearity, Accuracy, Response Factor: Linearity, accuracy and response factors should be established for the significant related substances during the method validation. In order to limit the workload of method development, usually less than 3 significant related substances should be selected in a method. Therefore, the other related substances should not be included in these experiments [71].

System suitability criteria: It is advisable to run system suitability tests in these robustness experiments. During the robustness testing of the method validation, critical method parameters such as mobile phase composition and column temperature are varied to mimic the day-to-day variability. Therefore, the system suitability results from these robustness experiments should reflect the expected range. Consequently, the limits for system suitability tests can be estimated from these experiments [72].

\section{Conclusion}

The optimized HPLC method described here provides fast and reliable analyses; it is a valuable tool during drug formulation development. Additionally, significant savings in time and money can be achieved. By designing chromatographic conditions to fit the needs of a specific experiment, analytical laboratories can save as much as $93 \%$ in time, relative to their original assays. Reproducible quality HPLC results can only be obtained if attention has been paid to the method development, validation and the system's suitability to carry out the analysis.

\section{References}

1. Liu SJ, Liu ZX, Ju WZ, Zhou L, Chen M, et al. (2010) Development and Validation of a Liquid Chromatographic/ Mass Spectrometric Method for the Determination of Saikosaponin a in Rat Plasma and its Application to Pharmacokinetic Study. J Anal Bioanal Techniques 1:104.

2. Ramadan A, Abd El-Aty AM (2011) Pharmacokinetics and Distribution of Florfenicol in Bronchial Secretions of Healthy and Pasteurella multocida Infected Calves. Pharm Anal Acta 2:117.

3. Anis SM, Hosny MM, Abdellatef HE, El-Balkiny MN (2011) Kinetic Spectrophotometric Determination of Betahistine Dihydrochloride and Etilefrine Hydrochloride in Pharmaceutical Formulation. Pharm Anal Acta 2:116.

4. Reddy YR, Kumar KK, Reddy MRP, Mukkanti K (2011) Rapid Simultaneous Determination of Sumatriptan Succinate and Naproxen Sodium in Combined Tablets by Validated Ultra Performance Liquid Chromatographic Method. J Anal Bioanal Techniques 2: 121.

5. Natesan S, Thanasekaran D, Krishnaswami V, Ponnusamy C (2011) Improved $\mathrm{Rp}$ - Hplc Method for the Simultaneous Estimation of Tranexamic Acid and Mefenamic Acid in Tablet Dosage Form. Pharm Anal Acta 2:115.

6. Puri A, Mehdi B, Panda NB, Dhawan GDPS (2011) Estimation of Pharmacokinetics of Propofol in Indian Pateints by HPLC Method. J Anal Bioanal Techniques 2: 120. 
7. Frag EYZ, Mohamed GG, Nour El-Dien FA, El-Badry Mohamed M (2011) The Use of Integrated Analytical Tools for Determination of Cyclopentolate and Naphazoline Hydrochlolrides in Pure and Pharmaceutical Preparations. Pharm Anal Acta 2: 114

8. Bari SB, Jain PS, Bakshi AR, Surana SJ (2011) HPTLC Method Validation for simultaneous determination of Tamsulosin Hydrochloride and Finasteride in Bulk and Pharmaceutical Dosage Form. J Anal Bioanal Techniques 2: 119.

9. Manassra A, Khamis M, el-Dakiky M, Abdel-Qader Z, Al-Rimawi F (2010) Simultaneous HPLC Analysis of Betamethasone and Clotrimazole in Cream Formulations. Pharm Anal Acta 1: 113

10. Choudhari VP, Nikalje AP (2010) Simultaneous Estimation of Atorvastatin Ezetimibe and Fenofibrate in Pharmaceutical Formulation by RP-LC-PDA Pharm Anal Acta 1: 111

11. Chitlange SS, Chaturvedi KK, Wankhede SB (2011) Development and Validation of Spectrophotometric and HPLC Method for the Simultaneous Estimation of Salbutamol Sulphate and Prednisolone in Tablet Dosage Form. J Anal Bioanal Techniques 2: 117.

12. Dias CL, Bajerski L, Rossi RC, Bergold AM, Fröehlich PE (2010) Comparative Validation Study to Assay Milnacipran Hydrochloride in Capsules by a StabilityIndicating LC and a Second Order Derivative UV Spectroscopic Methods. Pharm Anal Acta 1: 112

13. Maithani M, Singh R (2011) Development and Validation of a StabilityIndicating HPLC Method for the Simultaneous Determination of Salbutamo Sulphate and Theophylline in Pharmaceutical Dosage Forms. J Anal Bioanal Techniques 1: 116

14. Prabu SL, Srinivasan M, Thiagarajan S, Marina Q (2010) Simultaneous Determination of Gatifloxacin and Ambroxol Hydrochloride in a Tablet Formulation by Liquid Chromatography. Pharm Anal Acta 1: 110.

15. Babu ARS, Thippeswamy B, Vinod AB (2011) Determination of Tacrolimus in Rat Whole Blood Utilizing Triple Quadrupole LC/MS. J Anal Bioanal Techniques 2: 118.

16. Liu Y, Hou S, Wang L, Yang S (2010) Development and Validation of a Liquid Chromatography Method for the Analysis of Paromomycin Sulfate and its Impurities. J Anal Bioanal Techniques 1: 102.

17. Sakurada T, Zusi S, Kobayashi E, Satoh N, Ueda S (2010) Simultaneous Determination of Morphine, Morphine Glucuronides (M3G, M6G) and Oxycodone in Human Plasma by High-performance Liquid Chromatography. J Anal Bioanal Techniques 1: 101.

18. Fayyad MK, Misha AK, Yousef Al-Musaimi OI (2010) Effect of Temperature Wavelength, pH, Ion Pair Reagents and Organic Modifiers' Concentration on the Elution of Cystatin C. Stability of Mobile Phase. J Anal Bioanal Techniques 1: 103

19. Lee T, Amore TD (2011) Membrane Separation Theoretical and Applicable Considerations for Optimum Industrial Bioprocessing. J Bioprocess Biotechniq 1: $101 \mathrm{e}$.

20. Kumar NS, Nisha N, Nirmal J, Sonali N, Bagyalakshmi J (2011) HPLC Determination of Pitavastatin Calcium in Pharmaceutical Dosage Forms. Pharm Anal Acta 2: 119.

21. Bari SB, Bakshi AR, Jain PS, Surana SJ (2011) Application of UVSpectroscopy and First Order Derivative Method for Determination of Tamsulosin Hydrochloride in Bulk and Tablets. Pharm Anal Acta 2: 120

22. Devika GS, Sudhakar M, Venkateshwara Rao J (2011) Simultaneous Determination of Eprosartan Mesylate and Hydrochlorthiazide in Pharmaceutical Dosage form by Reverse Phase High Performance Liquid Chromatography. Pharm Anal Acta 2: 122.

23. Bari SB, Bakhshi AR, Jain PS, Surana SJ (2011) Development and Validation of Stability- Indicating Tlc-Densitometric Determination of Ropinirole Hydrochloride in Bulk and Pharmaceutical Dosage Form. Pharm Anal Acta 2: 125.

24. Whitman DA, Abbott V, Fregien K, Bowers LD (1993) Recent advances in highperformance liquid chromatography/mass spectrometry and high-performance liquid chromatography/tandem mass spectrometry: detection of cyclosporine and metabolites in kidney and liver tissue. Ther Drug Monit 15: 552-556.

25. Somagoni J, Reddy S, Koorelli S, Manda S, Yamsani MR (2011) Enantiomeric Separation and Determination of Stereospecific Drug Release from Marketed Racemic Amlodipine Besylate Tablets by HPLC. Pharm Anal Acta 2: 129.
26. Singh SP, Wahajuddin, Jain GK (2010) Determination of Curcumin in Rat Plasma by Liquid-liquid Extraction using LC-MS/MS with Electrospray Ionization: Assay Development, Validation and Application to a Pharmacokinetic Study. J Bioanal Biomed 2: 079-084

27. Kuchekar SR, Kundlik ML, Zaware BH (2010) Rapid and Specifi c Approach for Direct Measurement of Topiramate in Human Plasma by LCMS/ MS Application for Bioequivalence Study. J Bioanal Biomed 2: 107-112.

28. Franceschi L, Furlanut M (2010) A Simple and Sensisitive HPLC Method to Monitor Serum and Synovial Fluid Concentrations of Ketorolac in Reumathologic Patients. J Bioanal Biomed 2: 121-124.

29. Stevenson PG, Tarafder A, Guiochon G (2011) Comprehensive twodimensional chromatography with coupling of reversed phase high performance liquid chromatography and supercritical fluid chromatography. J Chromatogr $A$ 2: 11-21.

30. Gao H, Li N, Wang JY, Zhang SC, Lin G (2011) Definitive diagnosis of hepatic sinusoidal obstruction syndrome induced by pyrrolizidine alkaloids. J Dig Dis 13: 33-39.

31. Kassaye L, Hymete A, Bekhit AA, Genete G (2011) Validation of an HPLC method for the simultaneous determination of diminazene diaceturate and phenazone in injectable veterinary granules and bulk powders. Pak J Pharm Sci 25: 255-259

32. Khan MK, Khan MF, Mustafa G, Sualah M (2011) Comparison of HighPressure Liquid Chromatography and microbiological assay for determination of ciprofloxacin tablets in human plasma employed in bioequivalence and pharmacokinetics study. Pak J Pharm Sci 25: 81-88.

33. Bouva MJ, Sollaino C, Perseu L, Galanello R, Giordano PC, et al. (2011) Relationship between neonatal screening results by HPLC and the number of $\alpha$-thalassaemia gene mutations; consequences for the cut-off value. J Med Screen 18: 182-186.

34. Hewala I, El-Fatatry H, Emam E, Mabrouk M (2011) Development and application of a validated stability-indicating high-performance liquid chromatographic method using photodiode array detection for simultaneous determination of granisetron, methylparaben, propylparaben, sodium benzoate, and their main degradation products in oral pharmaceutical preparations. AOAC Int 94: 1447-1460.

35. Elkady EF, Abbas SS (2011) Development and validation of a reversedphase column liquid chromatographic method for the determination of five cephalosporins in pharmaceutical preparations. J AOAC Int 94: 1440-1446.

36. Martins SM, Wendling T, Gonçalves VM, Sarmento B, Ferreira DC (2011) Development and validation of a simple reversed-phase HPLC method for the determination of camptothecin in animal organs following administration in solid lipid nanoparticles. J Chromatogr B Analyt Technol Biomed Life Sci 2: 169-170.

37. Byrro RM, César IC, de Santana E Silva Cardoso FF, Mundim IM, Teixeira LD et al. (2011) A rapid and sensitive HPLC-APCI-MS/MS method determination of fluticasone in human plasma: Application for a bioequivalency study in nasal spray formulations. J Pharm Biomed Anal 11: 213-214.

38. Zeng A, Xing J, Wang C, Song J, Li C, et al. (2011) Simultaneous analysis and retention behavior of major isoflavonoids in Radix Puerariae lobatae and Radix Puerariae thomsonii by high performance liquid chromatography with cyclodextrins as a mobile phase modifier. Anal Chim Acta 712: 145-151.

39. Fabiano-Tixier AS, Elomri A, Blanckaert A, Seguin E, Petitcolas E, et al (2011) Rapid and Green Analytical Method for the Determination of Quinoline Alkaloids from Cinchona succirubra Based on Microwave-Integrated Extraction and Leaching (MIEL) Prior to High Performance Liquid Chromatography. Int Mol Sci 12: 7846-7860.

40. Scheibe A, Krantz L, Gleixner G (2012) Simultaneous determination of the quantity and isotopic signature of dissolved organic matter from soil water using high-performance liquid chromatography/isotope ratio mass spectrometry. Rapid Commun Mass Spectrom 26: 173-180.

41. Mohammadi B, Majnooni MB, Khatabi PM, Jalili R, Bahrami G (2011) 9-Fluorenylmethyl chloroformate as a fluorescence-labeling reagent for derivatization of carboxylic acid moiety of sodium valproate using liquid chromatography/tandem mass spectrometry for binding characterization: A human pharmacokinetic study. J Chromatogr B Analyt Technol Biomed Life Sci 9: 25-28.

42. Raymond Naxing Xu, Leimin Fan, Matthew J Rieser, Tawakol A El-Shourbagy 
Citation: Atanu Kumar J (2012) HPLC: Highly Accessible Instrument in Pharmaceutical Industry for Effective Method Development. Pharm Anal Acta 3:147. doi:10.4172/2153-2435.1000147

(2007) Recent advances in high-throughput quantitative bioanalysis by LCMS/MS. Journal of Pharmaceutical and Biomedical Analysis 44: 342-355.

43. Shintani H, Sakudo A (2011) HPLC Analysis and Identification of Compounds Inhibiting Bacterial Growth in Ozone Gas Sterilized Polysulfone and Polycarbonate. Pharm Anal Acta 2: 130

44. Jain R, Sahu V, Jain N, Jain S (2011) Mixed Hydrotropy Solubilization Approach for Quantitative Estimation of Eprosartan Mesylate and Hydrochlorthiazide by UV Spectrophotometer. Pharm Anal Acta 2: 135.

45. Dorottya Bartos, Sándor Görög (2008) Recent Advances in the Impurity Profiling of Drugs. Current Pharmaceutical Analysis 4: 215-230.

46. Srivastava B, Sharma BK, Uttam Singh B, Yashwant, Neha Sethi (2010) Ultra performance liquid chromatography (uplc) : a chromatography technique. International Journal of Pharmaceutical Quality Assurance 2:19-25.

47. Holme P, Payne K, Smith S, Spence M (2011) A generic bioanalytical method development strategy and fit-for-purpose qualification to support $\mathrm{hERG}$ in vitro sample analysis. Bioanalysis 3: 2739-2745.

48. Quintas LEM, Gram KRS, da Silveira GPE, Lopes DVS, Pôças ESC (2011) Pharmacokinetic Modifications and Drug-Drug Interactions in Clinical Monitoring of the Elderly: A Short Review. Pharm Anal Acta 2: 141.

49. Naveen Kumar Reddy G, Rajendra Prasad VVS, Maiti NJ, Nayak D, Prashan Kumar M (2011) Development and Validation of a Stability Indicating UPLC Method for Determination of Moxifloxacin Hydrochloride in Pharmaceutical Formulations. Pharm Anal Acta 2: 142.

50. Remsberg CM, Yáñez JA, Vega-Villa KR, Davies NM, Andrews PK, et al. (2010) HPLC-UV Analysis of Phloretin in Biological Fluids and Application to Pre-Clinical Pharmacokinetic Studies. J Bioremed Biodegrad 1: 101.

51. Ekeberg D, Norli HR, Stene C, Devle H, Bergaust L (2010) Identification of Brominated Flame Retardants in Sediment and Soil by Cyclohexane Extraction and Gas Chromatography Mass Spectrometry. J Chromatograph Separat Techniq 1: 102.

52. Musmade PB, Deshpande PB, Pathak SM, Adiga MNS, Bhat MK, et al. (2010) High Performance Liquid Chromatographic Method for the Determination of Clobetasol in Rat Plasma and its Application to Skin Penetration.J Bioanal Biomed 2: 001-007.

53. Bai L, Ma Z, Yang G, Yang J, Cheng J (2011) A Simple HPLC Method for the Separation of Colistimethate Sodium and Colistin Sulphate. J Chromatograph Separat Techniq 1: 105.

54. Yasuhara A, Tanaka Y, Makishima M, Suzuki S, Shibamoto T (2011) LC-MS Analysis of Low Molecular Weight Carbonyl Compounds As 2,4-Dinitrophenylhydrazones Using Negative Ion Mode Electronspray Ionization Mass Spectrometry. J Chromatograph Separat Techniq 2: 107.

55. Sultana N, Arayne MS, Naveed S (2011) RP-HPLC Method for Simultaneous Determination of Captopril and Diuretics: Application in Pharmaceutical Dosage Forms and Human Serum. J Chromatograph Separat Techniq 2: 109.

56. Haider M (2011) Development and Validation of a Stability Indicating HPLC Method for the Estimation of Butamirate Citrate and Benzoic Acid in Pharmaceutical Products. J Chromatograph Separat Techniq 2: 111.

57. Abdelkawy M, Metwaly F, El Raghy N, Hegazy M, Fayek N (2011) Simultaneous determination of Ambroxol Hydrochloride and Guaifenesin by HPLC, TLCSpectrodensitometric and multivariate calibration methods in pure form and in Cough Cold Formulations. J Chromatograph Separat Techniq 2: 112.

58. Jain PS, Khatal RN, Jivani HN, Surana SJ (2011) Development and Validation of TLC-densitometry Method for Simultaneous Estimation of Brimonidine tartrate and Timolol maleate in Bulk and Pharmaceutical Dosage Form. J Chromatograph Separat Techniq 2: 113.

59. Hewavitharana AK, Shaw PN, Ng YK, Fuerst JN (2009) Simple Screening Method for Staurosporine in Bacterial Cultures using Liquid ChromatographyTandem Mass Spectrometry. J Bioanal Biomed 1: 001- 004.

60. Moreno RA, Costal O, Brum Junior L, Sverdloff CE, Domingues CC, et al (2009) Cimetidine Quantification in Human Plasma by Highperformance Liquid Chromatography Coupled to Electrospray Ionization Tandem Mass Spectrometry. Application to a Comparative Pharmacokinetics Study. J Bioanal Biomed 1: 005-013.
61. Venkatesh DN, Sankar S, Meyyanathan SN, Muralidharan S, Shanmugam R et al. (2009) Bioavailability Studies on Developed ProchlorperazineMaleate Sustained Release Tablets by HPLC. J Bioanal Biomed 1: 054-057.

62. Zufía L, Aldaz A, Ibáñez N, Giráldez J (2010) Validation of an LC Method for Therapeutic Drug Monitoring of Voriconazole in Patients. J Bioanal Biomed 2: 035-043.

63. Mohammad A, Laeeq S, Moheman A (2010) Sodium Deoxycholate Micelles Activated Separation of Coexisting Five-nucleobases by High-performance Thin-layer Chromatography. J Bioanal Biomed 2: 055-059.

64. Moses PF, Muralidharan S, Rajan S, Nagarajan, Suresh B (2010) Simple and Validated Method for Estimation of Amlodipine by LC-MS (ESI) Using Healthy Indian Human Volunteers: and Evaluation of Pharmacokinetic Parameters. J Bioanal Biomed 2: 069-074.

65. Franceschi L, Furlanut M (2010) A Simple and Sensisitive HPLC Method to Monitor Serum and Synovial Fluid Concentrations of Ketorolac in Reumathologic Patients. J Bioanal Biomed 2: 121-124.

66. Jain T, Bhandari A, Ram V, Sharma S, Chaudhary RK, et al. (2011) Highperformance Liquid Chromatographic Method with Diode Array Detection for Quantification of Haloperidol Levels in Schizophrenic Patients During Routine Clinical Practice. J Bioanal Biomed 3: 008-012.

67. Akhlaq M, Khan GM, Wahab A, Khan A, Hussain A (2011) A simple highperformance liquid chromatographic practical approach for determination of flurbiprofen. J Adv Pharm Technol Res 2: 151-155.

68. Peer CJ, Spencer SD, Vandenberg DA, Pacanowski MA, Horenstein RB (2011) A sensitive and rapid ultra HPLC-MS/MS method for the simultaneous detection of clopidogrel and its derivatized active thiol metabolite in human plasma. J Chromatogr B Analyt Technol Biomed Life Sci 9: 23-25.

69. Gratz SR, Ciolino LA, Mohrhaus AS, Gamble BM, Gracie JM (2011) Screening and determination of polycyclic aromatic hydrocarbons in seafoods using QuEChERS-based extraction and high-performance liquid chromatography with fluorescence detection. J AOAC Int 94: 1601-1616.

70. Harp BP, Belai N, Barrows JN (2011) Ultra-performance liquid chromatographic determination of manufacturing intermediates and subsidiary colors in D\&C Red No. 34 and its lakes. J AOAC Int 94: 1548-1554.

71. Avula B, Shukla YJ, Wang YH, Khan IA (2011) Quantitative determination of pregnanes from aerial parts of Caralluma species using HPLC-UV and identification by LC-ESI-TOF. J AOAC Int. 94: 1383-1390.

72. Yi P, Liu L, Mei H, Zeng F, Huang Z, et al. (2011) Establishment of reference range of plasma amino acids for younger Chinese children by reverse phase HPLC. J Pediatr Endocrinol Metab 24: 733-738. 